2. Vilke GM, Smith AM, Ray LU, Steen PJ, Murrin PA, Chan TC: Airway obstruction in children aged less than 5 years: the prehospital experience. Prehosp Emerg Care 2004, 8:196-199.

Conflict of interest None declared. Funding None declared.

\section{REVISING EMS DISPATCH PROCEDURES TO MANAGE GROWING DEMAND IN VICTORIA, AUSTRALIA}

${ }^{1} \mathrm{E}$ Andrew* ${ }^{1,2}{ }^{2}$ Karen Smith. ${ }^{1}$ Ambulance Victoria; ${ }^{2}$ Monash University

\subsection{6/bmjopen-2017-EMSabstracts.4}

Aim The Advanced Medical Priority Dispatch (AMPDS) system is used to triage emergency medical calls in Victoria, Australia. However, the level of response assigned to each AMPDS Event Type (ET), which may include triage away from an emergency medical service (EMS) attendance, is determined by Ambulance Victoria. This study aimed to increase the proportion of low-acuity calls diverted to secondary triage and onto an alternative service provider (ASP), ultimately reducing EMS demand.

Methods A review of the level of EMS response assigned to AMPDS ET was conducted using research, audit and clinical expertise. Existing 'time-critical' and 'acute but not time-critical' ETs were assessed for suitability against the assigned level of EMS response and existing dispatch rules. An analysis of events occurring pre-reform (8 Feb-8 May 2016) and postreform (9 May-8 Aug 2016) was conducted.

Results A total of 105 'time-critical' ETs were assessed as suitable for downgrade to an 'acute' response, while 221 'acute' and 'time-critical' ETs were deemed suitable for diversion to secondary triage. The changes were implemented using staged approach, commencing in October 2015. The proportion of cases receiving a 'time-critical' EMS response decreased from $55.1 \%$ pre-reform to $48.9 \%$ post-reform $(\mathrm{p}<0.001)$. The proportion of emergency calls avoiding EMS dispatch increased from $8.8 \%$ pre-reform to $14.1 \%$ post-reform $(p<0.001)$. Of the cases diverted to secondary triage post-reform, $32.4 \%$ were referred to an ASP, $30.2 \%$ were referred to a non-emergency transport service and $41.8 \%$ were returned for EMS dispatch.

Conclusion This study provides a sound methodological approach for revising EMS dispatch protocols. Ongoing monitoring of the dispatch changes aims to identify areas that may benefit from further optimisation.

Conflict of interest None declared.

Funding None declared.

\section{MENTAL HEALTH-RELATED PRESENTATIONS TO EMERGENCY MEDICAL SERVICES IN VICTORIA, AUSTRALIA}

${ }^{1} \mathrm{E}$ Andrew*, ${ }^{*} \mathrm{R}$ Roggenkamp, ${ }^{1,2} \mathrm{Z}$ Nehme, ${ }^{1,2} \mathrm{~S}$ Cox, ${ }^{1,2} \mathrm{~K}$ Smith. ${ }^{1}$ Ambulance Victoria; ${ }^{2}$ Monash University

\subsection{6/bmjopen-2017-EMSabstracts.5}

Aim Recent increases in demand for mental health services have not been matched with community resources, resulting in increased demand on emergency medical services (EMS). We sought to describe the clinical profile, demand patterns and care provided to mental health patients accessing EMS in Victoria, Australia.

Methods We conducted a retrospective observational study of cases occurring between January and December 2015. Computer Aided Dispatch and electronic patient care record data were extracted from the Ambulance Victoria data warehouse. Characteristics of mental health presentations were described using descriptive statistics.

Results Of the total 504,676 EMS attendances, 48041 (9.5\%) were mental health-related. Additionally, 4708 mental healthrelated secondary triages were included, representing $6.6 \%$ of the Victorian secondary triage caseload. Emergency mental health patients were younger and more often female than other patients attended by EMS $(p<0.001)$. Most mental health patients were transported to hospital (74.4\%), however paramedics provided treatment to significantly fewer mental health patients than other emergency patients $(12.4 \%$ vs. $50.3 \%, \mathrm{p}<0.001)$. In mental health patients $\leq 15$ years, the most common presentations involved social/emotional issues, while for patients aged $\geq 65$ years, the most common presentation was anxiety. In patients undergoing secondary triage, $52.5 \%$ were frequent callers or anxiety presentations. A total of $27.7 \%$ of triaged patients were referred to an alternative service, while $24.6 \%$ were managed as per a care plan.

Conclusion For mental health-related cases, EMS were predominantly utilised as a transport mechanism rather than for active medical intervention. The role of alternative services in provision of care for mental health patients requires further investigation.

Conflict of interest None declared.

Funding None declared.

\section{CHARACTERISTICS OF THUNDERSTORM ASTHMA EMS ATTENDANCES IN VICTORIA, AUSTRALIA}

${ }^{1} \mathrm{E}$ Andrew* ${ }^{*}{ }^{1,2} \mathrm{Z}$ Nehme, ${ }^{1,2,3} \mathrm{~S}$ Bernard, ${ }^{1,2} \mathrm{~K}$ Smith. ${ }^{1}$ Ambulance Victoria; ${ }^{2}$ Monash University; ${ }^{3}$ The Alfred Hospital

\subsection{6/bmjopen-2017-EMSabstracts.6}

Aim Thunderstorm Asthma (TA) occurs when a dangerous mix of pollen and severe weather trigger acute respiratory distress symptoms in people with allergic rhinitis and asthma. We sought to describe the characteristics of patients attended by emergency medical services (EMS) during the largest global epidemic of TA which occurred in Melbourne on 21/11/2016. Methods A retrospective observational study of electronic EMS patient care records was conducted for all cases occurring during TA, between $1800 \mathrm{hrs}$ on $21 / 11 / 2016$ and 2359 hours on 22/11/2016 (30 hours). Results were compared with a standard comparator period defined as the seven days prior to the event $(14 / 11 / 2016$ to $20 / 11 / 2016)$.

Results EMS responded to 3631 cases during the TA event, compared with an average of 2419 cases per 30 hours during the comparator period. During TA, the final paramedic diagnosis was acute respiratory distress in $28.3 \%$ of patients (Asthma $=18.0 \%$, Shortness of Breath $=10.3 \%$ ), compared with $3.6 \%$ of patients during the comparator period (Asthma $=0.6 \%$, Shortness of Breath $=3.0 \%, \mathrm{p}<0.001$ ). Whilst there was an absolute increase in the number of time-critical cases, the proportion of acute respiratory illness patients considered time-critical after initial paramedic assessment remained stable between the two periods $(42.0 \%$ vs. $43.5 \%, \mathrm{p}=0.6)$. 